\title{
Prospective association between adherence to dietary recommendations and incident depressive symptoms in the French NutriNet-Santé cohort
}

\author{
Moufidath Adjibade $^{1 *}$, Cédric Lemogne ${ }^{2,3,4}$, Chantal Julia ${ }^{1,5}$, Serge Hercberg ${ }^{1,5}$, Pilar Galan ${ }^{1}$, \\ Karen E. Assmann ${ }^{1}$ and Emmanuelle Kesse-Guyot ${ }^{1}$ \\ ${ }^{1}$ Equipe de Recherche en Epidémiologie Nutritionnelle (EREN), Centre d'Epidémiologie et Statistiques Sorbonne Paris Cité, \\ National Conservatory of Arts and Crafts (Cnam), Institute for Health and Medical Research (INSERM) (U1153), Institute for \\ Agricultural Research (INRA) (U1125), COMUE Sorbonne Paris Cité, Université Paris 13, F-93017 Bobigny, France \\ ${ }^{2}$ Sorbonne Paris Cité, Faculté de Médecine, Université Paris Descartes, F-75006 Paris, France \\ ${ }^{3}$ AP-HP, Service de Psychiatrie de l'Adulte et du Sujet Agé, Hôpitaux Universitaires Paris Ouest, F-75015 Paris, France \\ ${ }^{4}$ Centre de Psychiatrie et Neurosciences, Institute for Health and Medical Research (INSERM, U894), F-75006 Paris, France \\ ${ }^{5}$ Département de Santé Publique, Hôpital Avicenne, F-93017 Bobigny, France
}

(Submitted 13 September 2017 - Final revision received 18 January 2018 - Accepted 9 March 2018 - First published online 23 May 2018 )

\begin{abstract}
A posteriori healthier dietary patterns and several nutrients have been associated with lower risks of depression in various studies; however, evidence is lacking with regard to the prospective association between adherence to nutritional recommendations (food-based and nutrientbased recommendations) and incident depression or depressive symptoms. In this study, we investigate such associations in the NutriNet Santé cohort. The study sample included 26225 participants (aged 18-86 years) who were initially free of depressive symptoms. Adherence to nutritional recommendations was measured by four scores namely modified French Programme National Nutrition Santé-Guideline Score (mPNNS-GS), Alternative Healthy Eating Index-2010 (AHEI-2010), Probability of Adequate Nutrient Intake Dietary Score (PANDiet) and Diet Quality Index-International (DQI-I), using non-consecutive dietary record data during the first 2 years of follow-up (mean number of recording days $=8, \mathrm{sD} 2$ ). Depressive symptoms were defined by a Center for Epidemiologic Studies Depression Scale (CES-D) score $\geq 17$ for men and $\geq 23$ for women. We used Cox proportional hazards models to estimate hazard ratios and $95 \%$ CI, modelling the dietary scores as standardised continuous variables and as tertiles. Over a mean follow-up of 6 years, we identified 2166 incident cases of depressive symptoms. All dietary scores with the exception of the AHEI-2010 were significantly inversely associated with incident depressive symptoms. In the fully adjusted model, an increase of $1 \mathrm{sD}$ in the mPNNS-GS, PANDiet and DQI-I was, respectively, associated with an 8\% (95\% CI 4, 13), 5\% $(95 \%$ CI 1, 9) and $9 \%(95 \%$ CI 5, 13) reduction in the risk of depressive symptoms. Overall, these findings suggest that diet in accordance with national or international guidelines could have beneficial effects with regard to mental health.
\end{abstract}

Key words: Mental health: Depression: Dietary scores: Nutritional recommendations

Depression is one of the most prevalent mental health disorders and a leading cause of disability worldwide. It has been associated with poor quality of life, physical decline, higher risk of premature death and a large economic burden ${ }^{(1,2)}$. In this context, depression is a major global public health problem, and reducing its prevalence by acting on associated modifiable lifestyle factors, including diet, is of major importance ${ }^{(3)}$.

The relationship between the overall quality of the diet and the risk of depression or depressive symptoms has been evaluated in several studies, mostly using a posteriori dietary patterns based on correlations in observed dietary data. Overall, healthier diets (characterised by high consumption of plant foods, whole grain products, olive oil and fish) have been associated with a decreased risk of depression or depressive symptoms, whereas unhealthy Western eating habits (characterised by high consumption of sweet and fatty products, processed meats and refined grains products) have been associated with an increased risk of depression or depressive symptoms ${ }^{(4-6)}$.

To prevent chronic diseases and to promote overall health, nutritional recommendations have been issued by health authorities, and adherence to these recommendations can be estimated using a priori-defined dietary indexes. In France, based on the recommendations of the Programme National Nutrition Santé $(\mathrm{PNNS})^{(7)}$ and on the national recommended dietary allowances ${ }^{(8)}$,

Abbreviations: AHEI-2010, Alternative Healthy Eating Index-2010; CES-D, Center for Epidemiologic Studies Depression Scale; CU, consumption unit; DQI-I, Diet Quality Index-International; mPNNS-GS, modified French Programme National Nutrition Santé-Guideline Score; EI, energy intake; PANDiet, Probability of Adequate Nutrient Intake Dietary Score.

* Corresponding author: M. Adjibade, fax +33 1483889 31, email m.adjibade@eren.smbh.univ-paris13.fr 
the Programme National Nutrition Santé-Guideline Score (PNNS-GS) was developed to measure adherence to dietary recommendations for the general population ${ }^{(9)}$, and the Probability of Adequate Nutrient Intake Dietary score (PANDiet) to measure compliance with the recommended nutrient intakes ${ }^{(10)}$. Besides those mentioned earlier, various a priori dietary scores have been developed to date, including different versions of the healthy eating index (HEI), the dietary quality index-international (DQI-I), the Dietary Approaches to Stop Hypertension and various scores measuring adherence to the Mediterranean Diet (MD) ${ }^{(11)}$. Among them, only scores reflecting adherence to the MD and the HEI have been widely studied in associations with depression. Overall, the studies that have investigated the association between adherence to the MD and depression or depressive symptoms showed a protective effect ${ }^{(12-18)}$. Other cross-sectional ${ }^{(19-24)}$ and some prospective studies ${ }^{(15,25-27)}$ have also evaluated the associations between different a priori dietary scores (measuring adherence to national dietary guidelines) and the risk of depression or depressive symptoms, but only one study has compared such associations across different diet quality scores ${ }^{(15)}$. Overall, the different versions of the $\mathrm{HEI}^{(15,19-22,24,27)}$ and the Australian Recommended Food Score ${ }^{(26)}$ were significantly inversely associated with the risk of depression or depressive symptoms, whereas the Ireland food pyramid recommendations ${ }^{(23)}$ were not significantly associated with the risk of depressive symptoms. The PNNS-GS was also significantly inversely associated with recurrent depressive symptoms ${ }^{(25)}$. Available studies investigating the association of the HEI, or modified versions, with the risk of depression were mostly cross-sectional, and few studies were based on non-American population. Thus, such relationships should be replicated in other settings.

In addition, several vitamins and minerals have been associated with depression in various studies ${ }^{(28,29)}$. However, most dietary scores do not include micronutrients, which may hinder the capacity of such scores to adequately reflect achievement of recommended nutrient intakes. To our knowledge, no study has yet examined the association between scores based on recommended nutrient intakes (e.g. PANDiet) or both recommended nutrient and food intakes (e.g. DQI-I) and the risk of depression or depressive symptoms. As nutritional recommendations were not primarily issued to prevent depression, further prospective studies using different nutritional scores are needed to examine whether some specific scores may perform better than others, thus informing preventive strategies.

In the present study, we thus aimed at investigating the prospective association between the overall quality of the diet measured by different scores reflecting adherence to food and nutrient recommendations, including the modified version of the PNNS-GS (mPNNS-GS), the Alternative Healthy Eating Index2010 (AHEI-2010), PANDiet and DQI-I and the risk of incident depressive symptoms in a large French prospective cohort.

\section{Methods}

\section{Study population}

We used data from the NutriNet-Santé study, a web-based observational cohort launched in France in 2009, which aims to investigate the relationship between nutrition and health, as well as the determinants of dietary behaviours and nutritional status. The design and methodology of the study have been described in detail elsewhere ${ }^{(30)}$. In brief, participants are adult volunteers (aged $\geq 18$ years) with internet access recruited from the general population by a vast multimedia campaign. Upon enrolment and each year thereafter, participants are asked to complete a set of self-administered web-based questionnaires assessing socio-demographic factors, economic conditions, physical activity, dietary intake, anthropometrics and health status. The NutriNet-Santé study is conducted in accordance with the Declaration of Helsinki and was approved by the ethics committee of the French Institute for Health and Medical Research (IRB Inserm no. 0000388FWA00005831) and by the National Commission on Informatics and Liberty (CNIL no. 908450 and no. 909216). Electronic informed consent was obtained from all participants. The NutriNet-Santé study is registered in ClinicalTrials.gov (NCT03335644).

\section{Depressive symptoms}

Depressive symptoms were assessed using the French version of the validated self-administered Center for Epidemiologic Studies Depression (CES-D) scale ${ }^{(31,32)}$ sent to all participants included in the NutriNet-Santé study two years after inclusion and every two years thereafter (with currently a maximum set of three completed CES-D questionnaires). The twenty items of the scale evaluate the frequency of depressive symptoms during the preceding week, using a four-point scale $(0=$ ' $<1 \mathrm{~d}$ '; $1=$ ' $1-2$ d'; $2=$ ' $3-4$ d'; and 3 = '5-7 d'). These are summed to yield a total score between 0 and 60 points, with higher scores denoting more depressive symptoms. The Cronbach's coefficient $\alpha$ (used to assess the internal consistency or reliability of a test or scale $\left.{ }^{(33)}\right)$ was $>0 \cdot 80$ for all three measures of the CES-D scale in our study, indicating good internal consistency.

The French validated cut-off value (CES-D $\geq 17$ in men and $\geq 23$ in women) was used to define the presence of depressive symptoms ${ }^{(32)}$. We defined incident cases of depressive symptoms as participants who were free of depressive symptoms at the first CES-D assessment and those who had depressive symptoms at least once during follow-up.

\section{Dietary data and dietary scores computation}

At enrolment and every 6 months thereafter, participants were invited to provide three non-consecutive dietary records, randomly assigned over a 2 -week period (2 weekdays and 1 weekend day). All foods and beverages consumed at each eating occasion were reported via a validated web-based dietary record tool designed for self-administration ${ }^{(34-36)}$. Portion sizes were indicated using validated photographs ${ }^{(37)}$, household measures or by indicating the exact quantity ( $\mathrm{g}$ ) or volume $(\mathrm{ml})$. Dietary data from the first 2 years of follow-up (corresponding to the time window between baseline and the 1st CES-D assessment) were used in the present study. For each participant, daily mean food consumption was calculated from all available dietary records (mean number of recording days $=8.03 ; \mathrm{SD}=2 \cdot 26$ ), weighted according to the type of day 
(weekdays or weekend). Energy and nutrient intakes were estimated using the NutriNet-Santé composition table including more than 3000 food items ${ }^{(38)}$. In addition, weekly fish and seafood intake was estimated by a self-administrated frequency questionnaire and alcohol intake (g ethanol/d) was estimated using an alcohol consumption frequency questionnaire when no consumption was reported in dietary records.

Dietary under-reporting was identified using the method developed by Black $^{(39)}$, which states that for an individual of stable weight energy intake (EI) and energy expenditure (EE) are equal. Thus, the ratio between EI and BMR (EI:BMR) is equivalent to a physical activity level (PAL) for which a minimum cut-off limit below which it is impossible to remain in stable weight has been defined ${ }^{(40)}$. The EI:BMR was thus calculated for each participant, and dietary under-reporters were defined as participants who reported average energy consumption so that the PAL was below the minimum cut-off. Average EI was calculated from the EI of all validated dietary records and BMR was estimated using Schofield equations based on sex, age, weight and height ${ }^{(41)}$. Two PAL cut-off limits were considered: a cut-off of 0.88 to identify the 'extreme' under-reporters who were systematically excluded and a cut-off of 1.55 for the remaining under-reporters. Of these, some participants who were identified as under-reporters were not considered as such (declaration of unusual consumption, diet for weight loss or recent weight loss $>5 \mathrm{~kg}$ ) and thus were not excluded

Overall diet quality was measured by using four different dietary scores including the mPNNS-GS, the AHEI-2010, the DQI-I and the PANDiet. The mPNNS-GS and AHEI-2010 mostly reflect food-based dietary guidelines ${ }^{(9,42)}$, the PANDiet reflects nutrient-based reference recommendations ${ }^{(10)}$ and the DQI-I reflects both food-based and nutrient-based recommendations $^{(43)}$. The computation of each dietary score has been extensively described elsewhere ${ }^{(9,10,42,43)}$. It is noteworthy that, for each score, points were assigned using predefined cut-off points (portion sizes or recommended nutrient intakes $)^{(9,10,42,43)}$. The description and scoring systems of the different scores are presented in Table 1 and detailed in the online Supplementary Material S1.

\section{Baseline covariates}

Socio-demographic data collected using the validated webbased questionnaire ${ }^{(44)}$ provided data on sex, date of birth, marital status (living alone, cohabiting or separated/divorced/ widowed), educational level (less than high school diploma, high school diploma or university level), occupational categories (never-employed/other activity, self-employed, employee, intermediate profession and managerial staff), residential area (rural or urban) and smoking status (never, former or current smoker). Monthly household income was also provided and estimated per consumption unit (CU) according to a weighting system where one $\mathrm{CU}$ is attributed for the first adult in the household, $0.5 \mathrm{CU}$ for other persons aged 14 years or older and $0.3 \mathrm{CU}$ for children under 14 years ${ }^{(45)}$. Categories of monthly household income per CU were defined as follows: $<1200,1200-1800,1800-2700,>2700$ euros and a category of participants who refused to disclose their income. Weight and height data were collected by a validated self-administered anthropometric questionnaire ${ }^{(46)}$ and BMI was calculated as the ratio of weight to squared height $\left(\mathrm{kg} / \mathrm{m}^{2}\right)$. Participants were classified as underweight or normal weight (BMI $<25 \mathrm{~kg} / \mathrm{m}^{2}$ ), overweight $\left(25 \leq \mathrm{BMI}<30 \mathrm{~kg} / \mathrm{m}^{2}\right)$ or obese $\left(\mathrm{BMI} \geq 30 \mathrm{~kg} / \mathrm{m}^{2}\right)^{(47)}$. Physical activity was assessed using a short form of the French version of the International Physical Activity Questionnaire ${ }^{(48)}$, a validated tool based on three specific types of physical activity: walking, activities of moderate intensity and activities of vigorous intensity. EE expressed in metabolic equivalent task minutes per week was estimated and classified as low physical activity ( $<30 \mathrm{~min}$ of physical activity; equivalent to brisk walking/d), moderate physical activity ( $\geq 30$ and $<60 \mathrm{~min}$ ) or high physical activity ( $\geq 60 \mathrm{~min}$ ), according to the French guidelines for physical activity $^{(9)}$. Prevalent and incident cases of cancer and CVD were self-reported during follow-up and validated by a medical committee. Type 2 diabetes was also self-reported.

In this study, data were missing for some covariates $(7$ for marital status, 70 for occupational categories, 309 for residential area, 191 for educational level and 411 for physical activity); however, the proportion of missing values was $<1 \%$ for all variables. Missing data for covariates were handled using the Hot Deck method, which consists of replacing the missing value with that of respondents with the same characteristics ${ }^{(49)}$.

\section{Statistical analysis}

Selection of the study sample. This study focused on participants who received at least two times the CES-D questionnaire (included between 2009 and 2011, $n$ 124925). Among the 35782 eligible participants for inclusion (participants with at least two completed CES-D questionnaires during follow-up and without depressive symptoms at the first CES-D measurement to ensure a prospective design), we included participants who had available data for computation of the dietary scores and who had not reported depression or treatment with antidepressants during the dietary data collection (before the first CES-D assessment). Thus, a final study sample of 25837 men and women was obtained (Fig. 1).

Characteristics of the participants. Participants included in this study were compared with excluded eligible participants using $\chi^{2}$ tests or $t$ tests as appropriate. Participants' characteristics and nutritional factors were compared across tertiles of the mPNNS-GS using linear contrast or Cochran-Mantel-Haenszel tests. For descriptive purposes, nutrient intakes were energyadjusted using the residual method ${ }^{(50)}$.

Statistical models. The associations between the mPNNS-GS, PANDiet, DQI-I and the AHEI-2010 (all modelled as tertiles to simplify the interpretation of results as low, medium and high adherence) and incident depressive symptoms were assessed using Cox proportional hazards regression models for interval censored data. Hazard ratios (HR) and $95 \%$ CI were estimated. Linear trend tests across the tertiles of dietary scores were performed by modelling the tertiles of dietary scores as ordinal 
Table 1. Description and the scoring system of the investigated dietary scores

\begin{tabular}{|c|c|c|c|}
\hline Dietary scores & Components & Scoring & Score range \\
\hline $\begin{array}{l}\text { Modified version of the } \\
\text { Programme National Nutrition } \\
\text { Santé-Guideline Score } \\
\text { (mPNNS-GS) }^{(9)} \\
\text { Based on French dietary } \\
\text { guidelines }\end{array}$ & $\begin{array}{l}\text { Food intake recommendations: fruit and vegetables without potatoes }(0-2) \text {, } \\
\text { starchy foods }(0-1) \text {, whole-grain products }(0-1) \text {, milk and dairy products } \\
(0-1) \text {, meat, poultry, fish, eggs and seafood }(0-1) \text {, seafood }(0-1) \text {, } \\
\text { vegetable fat }(0-1) \text {, water } v \text {. soda }(0-1) \\
\text { Moderation in consumption: sweetened foods }(-0.5-1) \text {, salt }(-0.5-1.5) \text {, } \\
\text { added fats }(0-2) \text {, alcohol }(0-1)\end{array}$ & $\begin{array}{l}\text { Physical activity component not included and points were } \\
\text { deducted if energy intake exceeded energy needs by } 5 \% \text {. } \\
\text { The mPNNS-GS score is the sum of components minus } \\
\text { penalty }\end{array}$ & $\begin{array}{l}0-13.5 \text { points, with } \\
\text { possibility of negative } \\
\text { values, owing to the } \\
\text { penalty system }\end{array}$ \\
\hline $\begin{array}{l}\text { Alternative Healthy Eating Index- } \\
2010 \text { (AHEI-2010)*(42) } \\
\text { Based on US dietary guidelines }\end{array}$ & $\begin{array}{l}\text { 'Desirable' components (contribute positively to the score; } 0-10 \text { points for } \\
\text { each component): vegetables without potatoes, fruit, whole grains, nuts } \\
\text { and legumes, long-chain } n-3 \text { fatty acids, PUFA and moderate alcohol } \\
\text { consumption } \\
\text { 'Undesirable' components (contribute negatively to the score; } 0-10 \text { points } \\
\text { for each component): Na, sugar-sweetened drinks and fruit juice, red } \\
\text { and processed meat }\end{array}$ & $\begin{array}{l}\text { Each component leads to a sub-score between } 0 \text { (less } \\
\text { healthy diet) and } 10 \text { (healthier diet), with intermediate } \\
\text { values scored proportionally to their intake. } \\
\text { The AHEI-2010 score is the sum of the components }\end{array}$ & $0-100$ points \\
\hline $\begin{array}{l}\text { Probability of Adequate Nutrient } \\
\text { Intake Dietary score } \\
\text { (PANDiet) })^{(10)} \\
\text { Based on adequate nutrient } \\
\text { intakes for the French } \\
\text { population }\end{array}$ & $\begin{array}{l}\text { Adequacy sub-score (0-100 points): Probability of adequate intake for } \\
\text { protein, total carbohydrate, total fat, PUFA, } n-3, n-6, n-3 \text {-DHA, } n-3 \text { - EPA- } \\
\text { DHA, fibre, vitamin } \mathrm{A} \text {, thiamin, riboflavin, niacin, folate, vitamin } \mathrm{B}_{5}, \mathrm{~B}_{6} \text {, } \\
\mathrm{B}_{12}, \mathrm{C}, \mathrm{D} \text { and } \mathrm{E}, \mathrm{I}, \mathrm{Se}, \mathrm{Ca}, \mathrm{Mg}, \mathrm{Zn}, \mathrm{P}, \mathrm{K}, \mathrm{Fe} \\
\text { Moderation sub-score }(0-100 \text { points): Probability of non-excessive intake } \\
\text { for protein, total carbohydrate, total fat, SFA, cholesterol, free sugars and } \\
\mathrm{Na} \\
\text { Penalty if intake exceeded the upper tolerable limit for retinol, niacin, folate, } \\
\text { vitamins } \mathrm{B}_{6}, \mathrm{C}, \mathrm{D}, \mathrm{E}, \mathrm{Ca}, \mathrm{Mg}, \mathrm{Zn}, \mathrm{P}, \mathrm{Fe}\end{array}$ & $\begin{array}{l}\text { Calculation of 'probability of adequacy' for each nutrient: } \\
\text { intake above minimum values for adequacy sub-score or } \\
\text { below maximum values for moderation sub-score } \\
\text { The PANDiet score is the average of the sub-scores }\end{array}$ & $0-100$ points \\
\hline $\begin{array}{l}\text { Diet Quality Index-International } \\
\left(\text { DQI-I) } \ddagger^{(43)}\right. \\
\text { Based on US dietary guidelines }\end{array}$ & $\begin{array}{l}\text { Variety ( } 0-20 \text { points): overall food group variety (meat/poultry/fish/eggs, } \\
\text { dairy products/beans, grain, fruit, vegetable; } 0-15) \text {, within-group variety } \\
\text { for protein source (meat, poultry, fish, dairy products, beans, eggs; } 0-5) \\
\text { Adequacy ( } 0-40 \text { points): vegetable group, fruit group, grain group, fibre, } \\
\text { protein, Fe, Ca and vitamin C ( } 0-5 \text { for each group) } \\
\text { Moderation ( } 0-30 \text { points): total fat, SFA, cholesterol, Na and empty energy } \\
\text { foods ( } 0-6 \text { for each group) } \\
\text { Overall balance (0-10 points): macronutrient ratio (carbohydrate:protein: } \\
\text { fat; } 0-6) \text {, fatty acid ratio (PUFA:MUFA:SFA; } 0-4)\end{array}$ & $\begin{array}{l}\text { The DQI-I score is the sum of the variety, adequacy, } \\
\text { moderation and overall balance sub-score }\end{array}$ & $0-100$ points \\
\hline
\end{tabular}

* We did not include trans-fatty acid intakes (not available in our study).

† Probability of adequate nutrient intake $F\left(\frac{\bar{y}-r}{\sqrt{\mathrm{SD} r^{2}+S D y^{2} / n}}\right), F$ (ranged from 0 to 1 , where 1 represents a $100 \%$ probability that the usual intake was adequate): 'Probnorm' function in SAS, $\bar{y}$ is the mean intake, sD ${ }^{2} y$ the day-to-day variability

of intake, $n$ the number of dietary record days, $r$ the nutrient reference value, $\mathrm{sD}^{2} r$ the interindividual variability.

₹ For nutrients included in the DQI-I computation, we used the recommended intakes for the French population (similar to those used in the PANDiet). 
124925 participants included in the NutriNet-Santé study who received at least two times the CES-D questionnaire (included between 2009 and 2012)

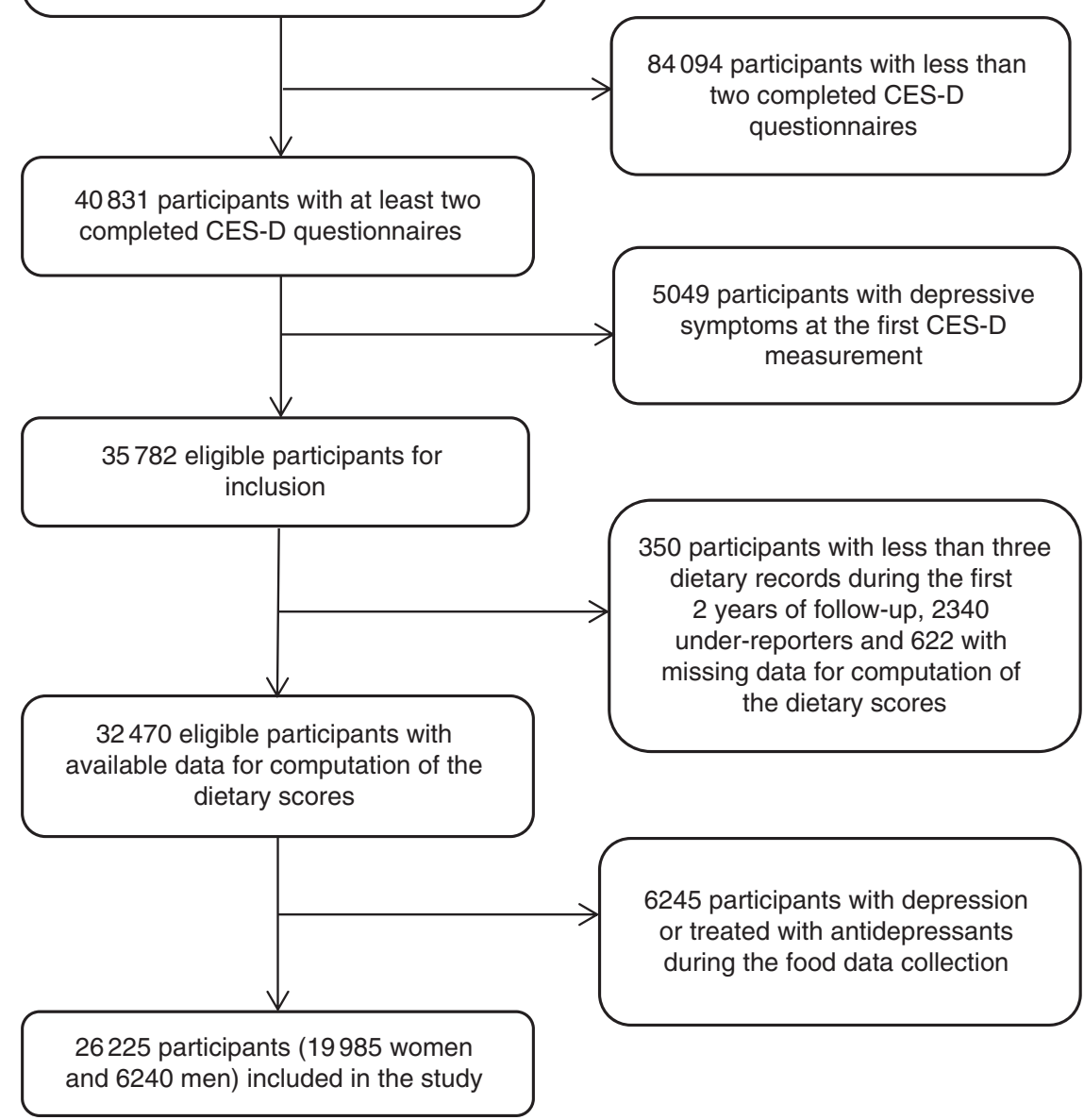

Fig. 1. Flow chart of participant selection. Center for Epidemiologic Studies Depression (CES-D) Scale.

variables. We also modelled the dietary scores as standardised continuous variables (individual score value minus the mean score value and divided by SD of the population) for comparison purposes. Age was used as the primary time-scale variable, with entry time defined as the age at the first CES-D measurement. For non-cases, exit time was defined as the age at last completed CES-D questionnaire, whereas for cases it was defined as the average of the age between the first occurrence of depressive symptoms and the age at the previous measurement ${ }^{(51)}$. The first model was adjusted for age, sex, marital status, educational level, occupational categories, monthly household income per CU, residential area, EI without alcohol, number of recording days and inclusion month. The second model was additionally adjusted for smoking status, physical activity and BMI (continuous variable). A final model (model 3) was performed to additionally account for cancer, type 2 diabetes and cardiovascular events during follow-up.

Sensitivity analyses. Supplementary analyses were performed to test the robustness of our findings. First, we evaluated the association between the dietary scores and incident depressive symptoms among the following: (A) participants who did not report treatment with anti-depressants during follow-up, as the use of anti-depressants could conceal the presence of depressive symptoms; and (B) participants who had completed $\geq 6 \mathrm{~d}$ of dietary records. Second, we defined the presence of depressive symptoms in both men and women by using a cut-off value of $16^{(31)}$, and an alternative cut-off value of nineteen validated for the French population ${ }^{(52)}$. All statistical analyses were conducted using SAS (version 9.4; SAS institute Inc.) with a significance level of 0.05 for two-sided tests.

\section{Results}

\section{Sample characteristics}

The study sample consisted of 19985 women and 6240 men with a mean age of 45.5 (SD 13.9) and 53.0 (SD 13.5) years, respectively, at inclusion. Compared with excluded participants ( $n$ 9557) from the eligible population (participants with at least two completed CES-D questionnaires during the follow-up and without depressive symptoms at the first 
CES-D measurement), included participants had higher levels of education, were more physically active, more likely to be men, co-habiting, managerial staff and never smokers. They were also more likely to have a BMI value in the normal-weight range, a household income per unit consumption $\geq 1800$ euros and less likely to have a chronic disease (online Supplementary Table S1).

During follow-up (mean follow-up $=5.9$ years, SD 1.2 ), we identified 2166 first cases of depressive symptoms. Baseline characteristics of our study population across tertiles of the mPNNS-GS are presented in Table 2. Participants with high adherence (tertile 3) compared with those with low adherence (tertile 1) were older, more physically active, less likely to live alone and more likely to be women, non-smokers, managerial staff or having an intermediate profession, to have a household income per unit consumption $\geq 1800$ euros and a chronic disease. In addition, a higher mPNNS-GS was associated with a higher EI from carbohydrates and proteins, higher intakes of total PUFA, fibre, most vitamins and minerals, but a lower EI from lipids and a lower intake of saturated and MUFA (Table 3). Similar associations were observed for the other scores examined in this study, with the exception of the AHEI-2010, which was positively associated with intakes of MUFA (online Supplementary Table S2).

Table 2. Baseline characteristics of 26225 participants according to the tertiles of the modified Programme National Nutrition Santé Guideline Score (mPNNS-GS), NutriNet-Santé study

(Numbers and percentages; mean values and standard deviations)

\begin{tabular}{|c|c|c|c|c|c|c|c|}
\hline \multirow[b]{2}{*}{ Baseline characteristics } & \multicolumn{2}{|c|}{ Tertile 1} & \multicolumn{2}{|c|}{ Tertile 2} & \multicolumn{2}{|c|}{ Tertile 3} & \multirow[b]{2}{*}{$P_{\text {trend }}{ }^{*}$} \\
\hline & $n$ & $\%$ & $n$ & $\%$ & $n$ & $\%$ & \\
\hline mPNNS-GS & \multicolumn{2}{|c|}{9014} & \multicolumn{2}{|c|}{8515} & \multicolumn{2}{|c|}{8696} & \\
\hline Range & \multicolumn{2}{|c|}{$0 \cdot 1-7 \cdot 5$} & \multicolumn{2}{|c|}{$7 \cdot 6-9 \cdot 0$} & \multicolumn{2}{|c|}{$9 \cdot 0-13 \cdot 0$} & \\
\hline Age (years) & & & & & & & $<0.0001$ \\
\hline Mean & \multicolumn{2}{|c|}{$43 \cdot 0$} & \multicolumn{2}{|c|}{$47 \cdot 2$} & \multicolumn{2}{|c|}{$51 \cdot 8$} & \\
\hline SD & \multicolumn{2}{|c|}{$14 \cdot 3$} & \multicolumn{2}{|c|}{$14 \cdot 0$} & \multicolumn{2}{|c|}{$12 \cdot 8$} & \\
\hline Sex $(\%)$ & & & & & & & $<0.0001$ \\
\hline Male & 2349 & $26 \cdot 1$ & 1994 & $23 \cdot 4$ & 1897 & $21 \cdot 8$ & \\
\hline Female & 6665 & 73.9 & 6521 & $76 \cdot 6$ & 6799 & $78 \cdot 2$ & \\
\hline Marital status (\%) & & & & & & & $<0.0001$ \\
\hline Living alone & 1447 & $16 \cdot 1$ & 1112 & $13 \cdot 0$ & 1004 & 11.6 & \\
\hline Co-habiting & 6817 & $75 \cdot 6$ & 6570 & $77 \cdot 2$ & 6584 & $75 \cdot 7$ & \\
\hline Separated/divorced/Widowed & 750 & $8 \cdot 3$ & 833 & $9 \cdot 8$ & 1108 & $12 \cdot 7$ & \\
\hline Educational level (\%) & & & & & & & 0.001 \\
\hline$<$ High school diploma & 1643 & $18 \cdot 2$ & 1612 & $18 \cdot 9$ & 1838 & $21 \cdot 1$ & \\
\hline High school diploma & 1513 & $16 \cdot 8$ & 1240 & $14 \cdot 6$ & 1302 & $15 \cdot 0$ & \\
\hline University level & 5858 & $65 \cdot 0$ & 5663 & $66 \cdot 5$ & 5556 & 63.9 & \\
\hline Occupational categories (\%) & & & & & & & $<0.0001$ \\
\hline Never-employed/other activity & 429 & $4 \cdot 8$ & 239 & 2.8 & 175 & $2 \cdot 0$ & \\
\hline Self-employed & 532 & $5 \cdot 9$ & 428 & $5 \cdot 0$ & 360 & $4 \cdot 1$ & \\
\hline Employee & 2469 & $27 \cdot 4$ & 2010 & $23 \cdot 6$ & 1934 & $22 \cdot 2$ & \\
\hline Intermediate profession & 2454 & $27 \cdot 2$ & 2438 & $28 \cdot 6$ & 2725 & 31.4 & \\
\hline Managerial staff & 3130 & $34 \cdot 7$ & 3400 & $39 \cdot 9$ & 3502 & $40 \cdot 3$ & \\
\hline Household income per consumption unit (\%) & & & & & & & $<0.0001$ \\
\hline Not answered & 938 & $10 \cdot 4$ & 803 & $9 \cdot 4$ & 858 & 9.9 & \\
\hline$<1200$ euros & 1419 & $15 \cdot 7$ & 1015 & 11.9 & 732 & $8 \cdot 4$ & \\
\hline $1200-1800$ euros & 2345 & $26 \cdot 0$ & 1949 & $22 \cdot 9$ & 1789 & $20 \cdot 6$ & \\
\hline 1800-2700 euros & 2214 & $24 \cdot 6$ & 2208 & 25.9 & 2273 & $26 \cdot 1$ & \\
\hline$\geq 2700$ euros & 2098 & $23 \cdot 3$ & 2540 & 29.9 & 3044 & $35 \cdot 0$ & \\
\hline Residential area (\%) & & & & & & & $<0.0001$ \\
\hline Rural & 2149 & $23 \cdot 8$ & 1884 & $22 \cdot 1$ & 1747 & $20 \cdot 1$ & \\
\hline Urban & 6865 & $76 \cdot 2$ & 6631 & $77 \cdot 9$ & 6949 & 79.9 & \\
\hline Smoking status (\%) & & & & & & & $<0.0001$ \\
\hline Never-smoker & 4574 & $50 \cdot 7$ & 4420 & 51.9 & 4556 & 52.4 & \\
\hline Former smoker & 2927 & 32.5 & 3158 & $37 \cdot 1$ & 3471 & 39.9 & \\
\hline Current smoker & 1513 & $16 \cdot 8$ & 937 & 11.0 & 669 & $7 \cdot 7$ & \\
\hline Physical activity (\%) & & & & & & & $<0.0001$ \\
\hline Low & 2753 & $30 \cdot 5$ & 2006 & $23 \cdot 6$ & 1476 & $17 \cdot 0$ & \\
\hline Moderate & 2181 & $24 \cdot 2$ & 2055 & $24 \cdot 1$ & 2014 & $23 \cdot 2$ & \\
\hline High & 4080 & $45 \cdot 3$ & 4454 & $52 \cdot 3$ & 5206 & 59.9 & \\
\hline BMI (\%) & & & & & & & 0.11 \\
\hline Underweight & 488 & $5 \cdot 4$ & 324 & 3.8 & 363 & $4 \cdot 2$ & \\
\hline Normal weight & 6010 & $66 \cdot 7$ & 5599 & $65 \cdot 8$ & 5767 & $66 \cdot 3$ & \\
\hline Overweight & 1811 & $20 \cdot 1$ & 1951 & 22.9 & 1993 & 22.9 & \\
\hline Obesity & 705 & $7 \cdot 8$ & 641 & $7 \cdot 5$ & 573 & $6 \cdot 6$ & \\
\hline Prevalence of cancer (\%) & 588 & $6 \cdot 5$ & 748 & $8 \cdot 8$ & 965 & $11 \cdot 1$ & $<0.0001$ \\
\hline Prevalence of CVD or type 2 diabetes (\%) & 2764 & $30 \cdot 7$ & 3006 & $35 \cdot 3$ & 3646 & 41.9 & $<0.0001$ \\
\hline
\end{tabular}

* $P_{\text {trend }}$ values are based on linear contrast or Cochran-Mantel-Haenszel tests. 
Table 3. Baseline nutritional factors of 26225 participants according to the tertiles of the modified Programme National Nutrition Santé Guideline Score (mPNNS-GS), NutriNet-Santé study

(Mean values and standard deviations)

\begin{tabular}{|c|c|c|c|c|c|c|c|}
\hline \multirow[b]{2}{*}{ Nutritional factors } & \multicolumn{2}{|c|}{ Tertile 1} & \multicolumn{2}{|c|}{ Tertile 2} & \multicolumn{2}{|c|}{ Tertile 3} & \multirow[b]{2}{*}{$P_{\text {trend }}{ }^{*}$} \\
\hline & Mean & SD & Mean & SD & Mean & SD & \\
\hline mPNNS-GS (range) & \multirow{2}{*}{\multicolumn{2}{|c|}{$\begin{array}{c}0.1-7.5 \\
9014\end{array}$}} & \multicolumn{2}{|c|}{$7 \cdot 6-9 \cdot 0$} & \multicolumn{2}{|c|}{$9 \cdot 0-13 \cdot 0$} & \\
\hline$n$ & & & & & & & \\
\hline Total energy intake $(\mathrm{kJ} / \mathrm{d})$ & 8485 & 2096 & 7837 & 1753 & 7502 & 1590 & $<0.0001$ \\
\hline Total energy intake $(\mathrm{kcal} / \mathrm{d})$ & 2028 & 501 & 1873 & 419 & 1793 & 380 & $<0.0001$ \\
\hline Alcohol intake $(g / d)$ & 11.0 & $14 \cdot 2$ & 8.4 & $10 \cdot 6$ & $6 \cdot 3$ & 7.5 & $<0.0001$ \\
\hline Energy intake without alcohol (kJ/d) & 8163 & 1996 & 7590 & 1691 & 7318 & 1536 & $<0.0001$ \\
\hline Energy intake without alcohol (kcal/d) & 1951 & 477 & 1814 & 404 & 1749 & 367 & $<0.0001$ \\
\hline Carbohydrates (\% energy) $\dagger$ & 42.6 & 5.9 & 43.0 & $5 \cdot 8$ & 44.0 & $5 \cdot 8$ & $<0.0001$ \\
\hline Lipids (\% energy) $\dagger$ & $40 \cdot 3$ & $5 \cdot 1$ & 38.8 & $5 \cdot 2$ & $37 \cdot 0$ & 5.5 & $<0.0001$ \\
\hline SFA $(g / d) \ddagger$ & 35.7 & 6.4 & $32 \cdot 9$ & $6 \cdot 0$ & $30 \cdot 1$ & 5.9 & $<0.0001$ \\
\hline MUFA $(\mathrm{g} / \mathrm{d}) \ddagger$ & 31.0 & $6 \cdot 0$ & $30 \cdot 6$ & $6 \cdot 0$ & 29.6 & 5.7 & $<0.0001$ \\
\hline PUFA $(g / d) \ddagger$ & $11 \cdot 0$ & $3 \cdot 4$ & 11.5 & 3.4 & 11.9 & 3.7 & $<0.0001$ \\
\hline$n-3$ Fatty acids $(\mathrm{g} / \mathrm{d}) \ddagger$ & 1.2 & 0.5 & 1.4 & 0.6 & 1.6 & 0.7 & $<0.0001$ \\
\hline Protein (\% energy) $\dagger$ & $16 \cdot 9$ & $3 \cdot 3$ & $17 \cdot 8$ & 3.4 & $18 \cdot 6$ & 3.4 & $<0.0001$ \\
\hline$\beta$-Carotene $(\mu \mathrm{g} / \mathrm{d}) \ddagger$ & 2857 & 1726 & 3588 & 1896 & 4332 & 2105 & $<0.0001$ \\
\hline Vitamin $C(\mathrm{mg} / \mathrm{d}) \ddagger$ & 99.1 & $55 \cdot 6$ & 118 & $61 \cdot 3$ & 141 & $65 \cdot 0$ & $<0.0001$ \\
\hline Vitamin D $(\mu \mathrm{g} / \mathrm{d}) \ddagger$ & 2.5 & 1.4 & $2 \cdot 7$ & 1.6 & 3.0 & 1.7 & $<0.0001$ \\
\hline Vitamin E $(\mathrm{mg} / \mathrm{d}) \ddagger$ & $10 \cdot 8$ & 3.3 & 11.7 & 3.2 & $12 \cdot 6$ & 3.2 & $<0.0001$ \\
\hline Folic acid $(\mu \mathrm{g} / \mathrm{d}) \ddagger$ & 290 & $72 \cdot 5$ & 332 & $76 \cdot 1$ & 381 & $85 \cdot 3$ & $<0.0001$ \\
\hline Vitamin $B_{12}(\mu \mathrm{g} / \mathrm{d}) \ddagger$ & 5.0 & 3.6 & 5.4 & 3.9 & $5 \cdot 8$ & 3.9 & $<0.0001$ \\
\hline $\mathrm{Mg}(\mathrm{mg} / \mathrm{d}) \ddagger$ & 305 & $374 \cdot 1$ & 340 & $78 \cdot 8$ & 382 & $87 \cdot 7$ & $<0.0001$ \\
\hline Fibre $(g / d) \ddagger$ & $16 \cdot 9$ & 4.4 & $10 \cdot 0$ & $4 \cdot 6$ & $23 \cdot 6$ & $5 \cdot 4$ & $<0.0001$ \\
\hline
\end{tabular}

* $P$ trend values are based on linear contrast.

$\dagger$ Values are percentages of total daily energy intake (without alcohol).

$\ddagger$ Values were adjusted for energy intake without alcohol using the residual method.

\section{Association between the dietary scores and incident depressive symptoms}

All dietary scores were significantly but rather modestly correlated with each other, with the highest correlation $(0 \cdot 71)$ found between the PANDiet and the DQI-I and the lowest (0.52) between the AHEI-2010 and the PANDiet (online Supplementary Table S3). The associations between the dietary scores and depressive symptoms are presented in Table 4. All dietary scores were inversely associated with incident depressive symptoms, but the association was non-significant for AHEI-2010. In the fully adjusted model, an increase of $1 \mathrm{sD}$ in the mPNNS-GS, PANDiet and DQI-I was, respectively, associated with an $8 \%(95 \% \mathrm{CI} 4,13, P=0.0002), 5 \%(95 \% \mathrm{CI} 1,9$, $P=0.02)$ and $9 \%(95 \% \mathrm{CI} 5,13, P<0.0001)$ reduction in the risk of depressive symptoms. When the scores were modelled as tertiles, the highest adherence to the mPNNS-GS, PANDiet and DQI-I was, respectively, associated with a $20 \%(95 \%$ CI 10, $28, P=0.0001), 12 \%$ (95\% CI $2,21, P=0.02)$ and $21 \%(95 \%$ CI $12,30, P<0 \cdot 0001)$ reduction in the risk of depressive symptoms compared with the lowest tertile.

\section{Sensitivity analyses}

In sensitivity analyses including only participants who were not treated with anti-depressants during follow-up, findings were not modified (online Supplementary Table S4). Applying a cut-off value of 16 or 19 to define depressive symptoms in both men and women did not substantially modify the observed associations with continuous standardised scores, but when the scores were evaluated in tertiles only the association between the mPNNS-GS and the DQI-I remained statistically significant in all models (online Supplementary Table S5 and S6). In addition, among the participants who completed $\geq 6 \mathrm{~d}$ of dietary records during the first 2 years of follow-up, only the mPNNS-GS and the DQI-I were significantly associated with a reduced risk of depressive symptoms (online Supplementary Table S7).

\section{Discussion}

In this large-scale longitudinal study, we investigated the association between adherence to nutritional recommendations (as measured by mPNNS-GS, AHEI-2010, PANDiet and DQI-I) and the risk of incident depressive symptoms over a 6-year followup period. We found a significant inverse association between adherence to the mPNNS-GS, the PANDiet and the DQI-I and the risk of depressive symptoms.

Regarding the AHEI-2010, which has been extensively used in the scientific literature, we found a non-significant association, in contrast to other published studies ${ }^{(15,19,24)}$. In the Seguimiento Universidad de Navarra Cohort study including 15093 participants, adherence to the AHEI-2010 at baseline was inversely associated with depression risk (HR quintile 5 compared with quintile $1: 0.72 ; 95 \%$ CI $0.59,0.88)^{(15)}$. In the cross-sectional studies published by Saneei et al. including 3363 Iranian adults with a mean age of $36.3(\mathrm{sD} 7.9)$ years, adherence to the AHEI-2010 was also significantly inversely associated with depression risk ${ }^{(19,24)}$. In addition, a study based on the Whitehall II cohort including 4215 participants aged 
Table 4. Association between the dietary scores and incident depressive symptoms in 26225 participants, NutriNet-Santé study (Hazard ratios $(\mathrm{HR})$ and $95 \% \mathrm{Cl}$ )

\begin{tabular}{|c|c|c|c|c|c|c|c|c|c|c|}
\hline & \multicolumn{2}{|c|}{ Tertile 1} & \multicolumn{2}{|c|}{ Tertile 2} & \multicolumn{2}{|c|}{ Tertile 3} & \multirow[b]{2}{*}{$P_{\text {trend }}$} & \multicolumn{2}{|c|}{ Continuous* } & \multirow[b]{2}{*}{$P \dagger$} \\
\hline & $\mathrm{HR}$ & $95 \% \mathrm{Cl}$ & $\mathrm{HR}$ & $95 \% \mathrm{Cl}$ & $\mathrm{HR}$ & $95 \% \mathrm{Cl}$ & & $\mathrm{HR}$ & $95 \% \mathrm{Cl}$ & \\
\hline \multicolumn{11}{|l|}{ mPNNS-GS } \\
\hline Range & \multicolumn{2}{|c|}{$0.1-7.5$} & \multicolumn{2}{|c|}{$7 \cdot 6-9 \cdot 0$} & \multicolumn{2}{|c|}{$9 \cdot 0-13 \cdot 0$} & & & & \\
\hline Model 1‡ & 1.00 & - & 0.88 & $0.80,0.98$ & 0.78 & $0.70,0.87$ & $<0.0001$ & 0.90 & $0.86,0.95$ & $<0.0001$ \\
\hline Model $2 \S$ & 1.00 & - & 0.90 & $0.81,0.99$ & 0.80 & $0.72,0.90$ & 0.0001 & 0.91 & $0.87,0.96$ & 0.0002 \\
\hline Model 3॥ & 1.00 & - & 0.90 & $0.81,0.99$ & 0.80 & $0.72,0.90$ & 0.0001 & 0.92 & $0.87,0.96$ & 0.0002 \\
\hline \multicolumn{11}{|l|}{ PANDiet } \\
\hline Range & \multicolumn{2}{|c|}{$36 \cdot 8-61 \cdot 5$} & \multicolumn{2}{|c|}{$61.5-68 \cdot 6$} & \multicolumn{2}{|c|}{$68 \cdot 6-95 \cdot 1$} & & & & \\
\hline Model 1 & 1.00 & - & 0.92 & $0.83,1.02$ & 0.86 & $0.77,0.95$ & 0.004 & 0.94 & $0.90,0.98$ & 0.003 \\
\hline Model 2 & 1.00 & - & 0.93 & $0.84,1.03$ & 0.89 & $0.80,0.99$ & 0.03 & 0.95 & $0.91,0.99$ & 0.02 \\
\hline Model 3 & 1.00 & - & 0.93 & $0.84,1.03$ & 0.88 & $0.79,0.98$ & 0.02 & 0.95 & $0.91,0.99$ & 0.02 \\
\hline \multicolumn{11}{|l|}{ DQI-I } \\
\hline Range & \multicolumn{2}{|c|}{$28 \cdot 4-58 \cdot 8$} & \multicolumn{2}{|c|}{$58 \cdot 8-64 \cdot 1$} & \multicolumn{2}{|c|}{$64 \cdot 1-87 \cdot 1$} & & & & \\
\hline Model 1 & 1.00 & - & 0.87 & $0.78,0.96$ & 0.76 & $0.68,0.85$ & $<0.0001$ & 0.90 & $0.86,0.94$ & $<0.0001$ \\
\hline Model 2 & 1.00 & - & 0.89 & $0.80,0.98$ & 0.79 & $0 \cdot 70,0.88$ & $<0.0001$ & 0.91 & $0.87,0.95$ & $<0.0001$ \\
\hline Model 3 & 1.00 & - & 0.89 & $0.80,0.98$ & 0.79 & $0.70,0.88$ & $<0.0001$ & 0.91 & $0.87,0.95$ & $<0.0001$ \\
\hline \multicolumn{11}{|l|}{ AHEI-2010 } \\
\hline Range & \multicolumn{2}{|c|}{$7 \cdot 1-43 \cdot 6$} & \multicolumn{2}{|c|}{$43 \cdot 6-54 \cdot 5$} & \multicolumn{2}{|c|}{$54.5-95.9$} & & & & \\
\hline Model 1 & 1.00 & - & 0.90 & $0.82,1.00$ & 0.91 & $0.81,1.02$ & 0.08 & 0.96 & $0.92,1.01$ & 0.09 \\
\hline Model 2 & 1.00 & - & 0.92 & $0.83,1.02$ & 0.95 & $0.85,1.06$ & 0.32 & 0.98 & $0.94,1.03$ & 0.40 \\
\hline Model 3 & 1.00 & - & 0.93 & $0.83,1.03$ & 0.96 & $0.86,1.07$ & 0.40 & 0.98 & $0.94,1.03$ & 0.50 \\
\hline
\end{tabular}

mPNNS-GS modified Programme National Nutrition Santé Guideline Score; PANDiet Probability of Adequate Nutrient intake Dietary score; DQI-I Diet Quality Index-International; AHEI-2010, Alternative Healthy Eating Index-2010.

* HR for the increase of $1 \mathrm{SD}$.

$\dagger P$ for linear relation (dietary score as a continuous variable).

‡ Adjusted for age, sex, marital status, educational level, occupational categories, household income per consumption unit, residential area, energy intake without alcohol, number of recording days and inclusion month.

$\S$ Adjusted for all variables in model 1 and smoking status, physical activity and BMI.

II Adjusted for all variables in model 2 and health events during follow-up (cancer, type 2 diabetes and cardiovascular events).

35-55 years at baseline reported a significant inverse association between high adherence to the AHEI and recurrent depressive symptoms only among women (OR for a 1-sD increase: $0.59 ; 95 \%$ CI $0.47,0.75)^{(27)}$. A possible explanation of our findings concerning the AHEI-2010 could be related to the cut-off values and the portions sizes used in the computation of AHEI-2010, which are different from those of French nutritional recommendations and possibly less discriminant in the French context owing to cultural specificities. For instance, the French nutritional guidelines recommend a daily intake of at least $385 \mathrm{~g}$ of $n-3$ fatty acids EPA + DHA and $5 \%$ of EI from PUFA, whereas the cut-off values used in the AHEI-2010 computation were $250 \mathrm{~g}$ and $10 \%$, respectively.

Besides these studies, only one other investigation has focused on the French food-based guidelines in relation to depressive symptoms. In this prospective study among 3328 participants (baseline mean age of 49.5 years, sD 6.2 and mean follow-up=13years) from the French Supplémentation en Vitamines et Minéraux AntioXydants cohort, a 1-point increase in the mPNNS-GS was associated with a $13 \%$ (95\% CI 6, 20) reduction in the risk of chronic or recurrent depressive symptoms ${ }^{(25)}$, in agreement with the reduced risk of incident depressive symptoms found in our study. Our findings are also in agreement with the findings of the Australian Longitudinal Study on Women's Health, in which maintaining a moderate or high adherence to the Australian Recommended Food Score (including seven food group components; vegetables, fruit, protein foods, grains, dairy products, fats and alcohol) over a 6-year period was, respectively, associated with a 6\% (95\% CI
$1,20)$ and $14 \%(95 \%$ CI $4,23 \%)$ reduction in the risk of depression $^{(26)}$ among 7877 participants aged 50-55 years at baseline. To our knowledge, no previous study has directly investigated the association between adherence to nutrientbased recommendations such as the PANDiet or the DQI-I and the risk of depression or depressive symptoms.

Higher adherence to the dietary scores considered in this study reflects a high intake of various vitamins and minerals (generally provided by high consumption of whole-grain products, fruits, vegetables and fish) associated with a reduced risk of several diseases ${ }^{(53-55)}$. However, these dietary scores show some slight differences in terms of included components, cut-off values and scoring, leading to different nutrient intakes in tertiles, which could explain the observed differential associations with the risk of depressive symptoms. The main difference is that the mPNNS-GS and AHEI-2010 include mostly food-based components, whereas the PANDiet includes only nutrients. Finally, the DQI-I was specially developed to facilitate cross-country comparisons and includes both food-based components and nutrients. Comparing the mPNNS-GS, the AHEI-2010 and the DQI-I, only the mPNNS-GS includes a system of penalty for energy overconsumption and only the DQI-I takes into account food variety, proportionality in energy sources and fatty acid composition. In addition, the DQI-I and PANDiet do not take into account alcohol consumption. These disparities are illustrated by correlation coefficients, which are not very high $(<0 \cdot 80)$, although all these dietary scores have the same overall objective, which is to measure the nutritional quality of the diet. 
Some limitations of our study should be noted. Given the observational design of our study, we cannot entirely exclude reverse causality although the design is prospective. Second, despite a wide range of confounders included in our statistical models, unmeasured factors related to depression such as personality traits, family history of depressive disorders, stressful life events and sleep disorders ${ }^{(56,57)}$ might have led to potential residual confounding. Third, participants of the NutriNet-Santé study were volunteers in a nutritional cohort and thus more interested in nutritional issues and healthy lifestyles than the general population. Thus, any generalisation of our findings should be done with caution. Another limitation of our study pertains to the large proportion of participants excluded from the eligible population, which could include a potential bias in the risk estimates. However, we have elected to prioritise the accuracy of the dietary data and to reduce potential reverse causality phenomenon owing to depressive episode during the dietary data assessment. Finally, data on trans-fatty acids were not available in our study, which did not allow to fully compute the original AHEI-2010. Important strengths of this study include the prospective design of our study, its large sample of participants aged 18-86 years and without depression at the beginning, the repeated data assessment of depressive symptoms using a validated tool and the quality of the dietary data based on repeated dietary records, yielding a particularly high accuracy of intake estimations. The wide range of confounding factors also helped to improve the estimations

To conclude, our study showed that high adherence to food-based and nutrient-based national or international nutritional recommendations was associated with a reduced risk of incident depressive symptoms. Overall, our findings suggest that nutritional recommendations, although designed mostly for the prevention of nutrition-related chronic conditions such as obesity, cardiovascular disorders and cancer, are also associated with a reduction in the risk of depressive symptoms. Thus, promoting better adherence to national dietary guidelines, as well as adequate nutrient intakes, may be useful in a primary prevention strategy of depressive symptoms through modifiable factors in the general population. The results found in this study should be confirmed by further prospective studies.

\section{Acknowledgements}

The authors thank all the scientists, dietitians, technicians and assistants for their technical contribution to the NutriNet-Santé study. The authors especially thank Younes Esseddik, Thi Duong Van, Frédéric Coffinieres, Mac Rakotondrazafy, Régis Gatibelza and Paul Flanzy (computer scientists), and Nathalie Arnault, Véronique Gourlet, Dr Fabien Szabo, Julien Allegre, Anouar Nechba and Laurent Bourhis (data-manager/ biostatisticians). The authors also thank all the volunteers of the NutriNet-Santé cohort.

The NutriNet-Santé Study is supported by the French Ministry of Health (DGS), the French Public Health Agency, the French National Institute for Health and Medical Research (INSERM), the Medical Research Foundation (FRM), the French National Institute for Agricultural Research (INRA), the National Conservatory for Arts and Crafts (CNAM), the National Institute for Prevention and Health Education (INPES) and the Paris 13 University. M. A. was supported by a doctoral fellowship from the Ecole Doctorale Galilée, Paris 13 University, Sorbonne Paris Cité.

The author contributions were as follows: C. J., S. H., P. G. and E. K.-G. were responsible for the development of the design and protocol of the study; M. A. performed the statistical analysis and wrote the paper; E. K.-G. provided methodological guidance; M. A., C. L., C. J., S. H., P. G., K. E. A. and E. K.-G. were involved in interpreting the results and editing the manuscript for important intellectual content. All authors read and approved the final manuscript.

C. L. has received honoraria for board membership from Lundbeck and for speaking at invited symposia from Daiichi-Sankyo, Janssen, Lundbeck, Otsuka Pharmaceuticals and Servier. None of the other authors has any conflicts of interest to declare.

\section{Supplementary material}

For supplementary material/s referred to in this article, please visit https://doi.org/10.1017/S0007114518000910

\section{Reference}

1. World Health Organization (2013) Mental Health Action Plan 2013-2020. Geneva: WHO.

2. Doris A, Ebmeier K \& Shajahan P (1999) Depressive illness. Lancet 354, 1369-1375.

3. World Health Organization (2004) Global Strategy on Diet, Physical Activity and Health. Geneva: WHO.

4. Rahe C, Unrath M \& Berger K (2014) Dietary patterns and the risk of depression in adults: a systematic review of observational studies. Eur J Nutr 53, 997-1013.

5. Lai JS, Hiles S, Bisquera A, et al. (2014) A systematic review and meta-analysis of dietary patterns and depression in community-dwelling adults. Am J Clin Nutr 99, 181-197.

6. Li Y, Lv MR, Wei YJ, et al. (2017) Dietary patterns and depression risk: a meta-analysis. Psychiatry Res 253, 373-382.

7. Hercberg S, Chat-Yung S \& Chaulia M (2008) The French National Nutrition and Health Program: 2001-2006-2010. Int J Public Health 53, 68-77.

8. Martin A (2001) Apports nutritionnels conseillés pour la population Française (Recommended Dietary Allowances for the French Population), 3rd ed. Paris: Tec \& Doc, Lavoisier.

9. Estaquio C, Kesse-Guyot E, Deschamps V, et al. (2009) Adherence to the French Programme National Nutrition Sante Guideline Score is associated with better nutrient intake and nutritional status. J Am Diet Assoc 109, 1031-1041.

10. Verger EO, Mariotti F, Holmes BA, et al. (2012) Evaluation of a diet quality index based on the probability of adequate nutrient intake (PANDiet) using national French and US dietary surveys. PLOS ONE 7, e42155.

11. Arvaniti F \& Panagiotakos DB (2008) Healthy indexes in public health practice and research: a review. Crit Rev Food Sci Nutr 48, 317-327.

12. Psaltopoulou $\mathrm{T}$, Sergentanis $\mathrm{TN}$, Panagiotakos $\mathrm{DB}$, et al. (2013) Mediterranean diet, stroke, cognitive impairment, and depression: a meta-analysis. Ann Neurol 74, 580-591. 
13. Skarupski KA, Tangney CC, Li H, et al. (2013) Mediterranean diet and depressive symptoms among older adults over time. J Nutr Health Aging 17, 441-445.

14. Lai JS, Oldmeadow C, Hure AJ, et al. (2016) Longitudinal diet quality is not associated with depressive symptoms in a cohort of middle-aged Australian women. Br J Nutr 115, 842-850.

15. Sanchez-Villegas A, Henriquez-Sanchez $\mathrm{P}$, Ruiz-Canela M, et al. (2015) A longitudinal analysis of diet quality scores and the risk of incident depression in the SUN Project. BMC Med 13, 197.

16. Sanchez-Villegas A, Martinez-Gonzalez MA, Estruch R, et al. (2013) Mediterranean dietary pattern and depression: the PREDIMED randomized trial. BMC Med 11, 208-211.

17. Rienks J, Dobson AJ \& Mishra GD (2013) Mediterranean dietary pattern and prevalence and incidence of depressive symptoms in mid-aged women: results from a large communitybased prospective study. Eur J Clin Nutr 67, 75-82.

18. Adjibade M, Assmann KE, Andreeva VA, et al. (2018) Prospective association between adherence to the Mediterranean diet and risk of depressive symptoms in the French SU.VI. MAX cohort. Eur J Nutr 57, 1225-1235.

19. Saneei P, Hajishafiee M, Keshteli AH, et al. (2016) Adherence to Alternative Healthy Eating Index in relation to depression and anxiety in Iranian adults. Br J Nutr 116, 335-342.

20. Loprinzi PD \& Mahoney S (2014) Concurrent occurrence of multiple positive lifestyle behaviors and depression among adults in the United States. J Affect Disord 165, 126-130.

21. Beydoun MA, Fanelli Kuczmarski MT, Beydoun HA, et al. (2010) The sex-specific role of plasma folate in mediating the association of dietary quality with depressive symptoms. J Nutr 140, 338-347.

22. Kuczmarski MF, Cremer SA, Hotchkiss L, et al. (2010) Higher Healthy Eating Index-2005 scores associated with reduced symptoms of depression in an urban population: findings from the Healthy Aging in Neighborhoods of Diversity Across the Life Span (HANDLS) study. J Am Diet Assoc 110, 383-389.

23. Meegan AP, Perry IJ \& Phillips CM (2017) The Association between Dietary Quality and Dietary Guideline Adherence with Mental Health Outcomes in Adults: A Cross-Sectional Analysis. Nutrients 9, E238.

24. Saneei P, Esmaillzadeh A, Keshteli AH, et al. (2016) Combined healthy lifestyle is inversely associated with psychological disorders among adults. PLOS ONE 11, e0146888.

25. Collin C, Assmann KE, Andreeva VA, et al. (2016) Adherence to dietary guidelines as a protective factor against chronic or recurrent depressive symptoms in the French SU.VI. MAX cohort. Prev Med 91, 335-343.

26. Lai JS, Hure AJ, Oldmeadow C, et al. (2017) Prospective study on the association between diet quality and depression in mid-aged women over 9 years. Eur J Nutr 56, 273-281.

27. Akbaraly TN, Sabia S, Shipley MJ, et al. (2013) Adherence to healthy dietary guidelines and future depressive symptoms: evidence for sex differentials in the Whitehall II study. Am J Clin Nutr 97, 419-427.

28. Lai J, Moxey A, Nowak G, et al. (2012) The efficacy of zinc supplementation in depression: systematic review of randomised controlled trials. J Affect Disord 136, e31-e39.

29. Lim SY, Kim EJ, Kim A, et al. (2016) Nutritional factors affecting mental health. Clin Nutr Res 5, 143-152.

30. Hercberg S, Castetbon K, Czernichow S, et al. (2010) The Nutrinet-Sante Study: a web-based prospective study on the relationship between nutrition and health and determinants of dietary patterns and nutritional status. BMC Public Health 10, 242

31. Radloff LS (1977) The CES-D Scale, a self-report depression scale for research in the general population. Appl Psychol Meas 1, 385-401.
32. Führer R \& Rouillon F (1989) The French version of the Center for Epidemiologic Studies-Depression Scale. Psychiatrie et Psychologie 4, 163-166.

33. Cronbach LJ (1951) Coefficient alpha and the internal structure of tests. Psychometrika 16, 297-334.

34. Touvier M, Kesse-Guyot E, Mejean C, et al. (2011) Comparison between an interactive web-based self-administered $24 \mathrm{~h}$ dietary record and an interview by a dietitian for large-scale epidemiological studies. Br J Nutr 105, 1055-1064.

35. Lassale C, Castetbon K, Laporte F, et al. (2016) Correlations between fruit, vegetables, fish, vitamins, and fatty acids estimated by web-based nonconsecutive dietary records and respective biomarkers of nutritional status. J Acad Nutr Diet 116, 427-438.

36. Lassale C, Castetbon K, Laporte F, et al. (2015) Validation of a Web-based, self-administered, non-consecutive-day dietary record tool against urinary biomarkers. Br J Nutr 113, 953-962.

37. Le Moullec N, Deheeger M., Preziosi P, et al. (1996) Validation du manuel photos utilisé pour l'enquête alimentaire de l'étude SU.VI.MAX (Validation of the photo manual used for the dietary assessment of the SU.VI.MAX study). Cab Nutr Diet 31, 158-164

38. NutriNet-Santé Etude (2013) Table de Composition des Aliments de l'étude NutriNet-Santé (NutriNet-Santé Study Food Composition Database). Paris: Economica.

39. Black AE (2000) Critical evaluation of energy intake using the Goldberg cut-off for energy intake:basal metabolic rate. A practical guide to its calculation, use and limitations. Int J Obes Relat Metab Disord 24, 1119-1130.

40. Goldberg GR, Black AE, Jebb SA, et al. (1991) Critical evaluation of energy intake data using fundamental principles of energy physiology: 1 . Derivation of cut-off limits to identify under-recording. Eur J Clin Nutr 45, 569-581.

41. Schofield WN (1985) Predicting basal metabolic rate, new standards and review of previous work. Hum Nutr Clin Nutr 39, Suppl. 1, 5-41, 5-41.

42. Chiuve SE, Fung TT, Rimm EB, et al. (2012) Alternative dietary indices both strongly predict risk of chronic disease. J Nutr 142, 1009-1018.

43. Kim S, Haines PS, Siega-Riz AM, et al. (2003) The Diet Quality Index-International (DQI-I) provides an effective tool for cross-national comparison of diet quality as illustrated by China and the United States. J Nutr 133, 3476-3484.

44. Vergnaud AC, Touvier M, Mejean C, et al. (2011) Agreement between web-based and paper versions of a sociodemographic questionnaire in the NutriNet-Sante study. Int $J$ Public Health 56, 407-417.

45. Institut National de la Statistique et des Etudes Economiques (2016) Définition des Unités de Consommation (Consumption Units Definition). https://www.insee.fr/fr/metadonnees/definition/ c1802 (accessed April 2017).

46. Lassale C, Peneau S, Touvier M, et al. (2013) Validity of webbased self-reported weight and height: results of the NutrinetSante study. J Med Internet Res 15, e152.

47. World Health Organization (1995) Physical status: the use and interpretation of anthropometry. Report of a WHO Expert Committee. World Health Organ Tech Rep Ser $\mathbf{8 5 4}$ $1-452$.

48. Craig CL, Marshall AL, Sjostrom M, et al. (2003) International physical activity questionnaire: 12-country reliability and validity. Med Sci Sports Exerc 35, 1381-1395.

49. Andridge RR \& Little RJ (2009) The use of sample weights in hot deck imputation. J Off Stat 25, 21-36.

50. Willett W \& Stampfer MJ (1986) Total energy intake: implications for epidemiologic analyses. Am J Epidemiol 124, $17-27$. 
51. Finkelstein DM (1986) A proportional hazards model for interval-censored failure time data. Biometrics $\mathbf{4 2}$ 845-854.

52. Morin AJ, Moullec G, Maiano C, et al. (2011) Psychometric properties of the Center for Epidemiologic Studies Depression Scale (CES-D) in French clinical and nonclinical adults. Rev Epidemiol Sante Publique 59, 327-340.

53. Mikkelsen K, Stojanovska L, Prakash M, et al. (2017) The effects of vitamin $\mathrm{B}$ on the immune/cytokine network and their involvement in depression. Maturitas 96 , $58-71$.

54. Grosso G, Galvano F, Marventano S, et al. (2014) Omega-3 fatty acids and depression: scientific evidence and biological mechanisms. Oxid Med Cell Longev 2014, 313570 .

55. Miki T, Kochi T, Eguchi M, et al. (2015) Dietary intake of minerals in relation to depressive symptoms in Japanese employees: the Furukawa Nutrition and Health Study. Nutrition 31, 686-690.

56. Berk M, Williams LJ, Jacka FN, et al. (2013) So depression is an inflammatory disease, but where does the inflammation come from? BMC Med 11, 200-211.

57. Lopresti AL, Hood SD \& Drummond PD (2013) A review of lifestyle factors that contribute to important pathways associated with major depression: diet, sleep and exercise. J Affect Disord 148, 12-27. 